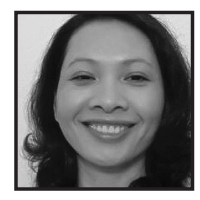

\title{
Rediscovering the Self Through Self-Reflection and Transformative Learning
}

\author{
Vanden Thong, Texas Christian University
}

\begin{abstract}
This article explores the relevancy and potential benefits of self-reflection and reflective practice in promoting personal and professional development. In addition, it reviews the concept of transformative learning in conjunction with self-reflection since the two constructs connect to each other as well as to the process of human development. Moreover, different educational programs and activities that increase the likelihood of change and transformation are discussed. Lastly, this article concludes with my personal reflections on my learning and growth as I adapted to change and navigated new environments while studying abroad in Great Britain.
\end{abstract}

\section{Shared Humanity}

II human beings will struggle with their mortality, experience
disappointments, and encounter unpredictable problems. How people
work through their emotions, manage their thoughts, and respond to different situations can determine whether they remain psychologically stagnant or find new ways of feeling, thinking, and behaving. The steps that people take to obtain new knowledge and grow as human beings vary widely. Each person's path appears unique. However, a broader perspective reveals the presence of a "shared humanity" and existence of universal experiences that invisibly unite all humanity (Silverman, 2008). Universal experiences and emotions related to doubt, fear, and loss transcend professional boundaries and individual differences. Regardless of the profession, 
everybody is in the process of becoming, including educators (Silverman, 2008). Palmer (1998) believed that the act of teaching, "tugs at the heart, opens the heart, [and] breaks the heart" (p. 11).

Learners and educators, patients and doctors, and clients and counselors possess this "shared humanity" and "universality" (Kottler, 2002; Rosin, 2015; Silverman, 2008). Everybody will encounter unplanned events and situations that can serve as openings for them to question who they are personally and professionally. These openings may allow individuals to examine the gaps in their lives, reconnect with their career passion, and rebuild their identity (Morgan, 2010). The individuals' state of mind and purposeful intention will affect their learning and growth (Morgan, 2010; Palmer \& Zajonc, 2010; Schön, 1987). This article explores the relevancy and potential benefits of self-reflection and reflective practice in promoting student growth and professional development. In addition, it reviews the concept of transformative learning in conjunction with selfreflection since the two constructs connect to each other and influence both education and human development. Moreover, I also discuss possible activities that are more likely to foster change and transformation. Lastly, this article concludes with my personal reflections while studying abroad in Great Britain.

\section{The Value of Self-Reflection}

Authors such as Paulo Freire, Parker Palmer, Jack Mezirow, and Donald Schön helped to explain the nature of human beings while providing insights into the learning process and the field of education (Freire \& Freire, 1994; Mezirow, 1991; Palmer, 1998; Schön, 1983, 1991). With greater insights about the learning process and change in teaching approach, a shift has occurred. Educators are transitioning from the teachercentered classroom model to a student-centered approach, which focuses more on self-reflection, self-understanding, and transformative education (Palmer \& Zajonc, 2010; Schön, 1983; Silverman, 2008). In addition, a greater number of scholarly literature is studying the link between emotional and cognitive growth and self-reflection. The use of reflective practice is contributing to the knowledge of transformative learning (Illeris, 2014; Kinsella, 2007; Morgan, 2010; Palmer \& Zajonc, 2010; Schön, 1983; StevensLong, Schapiro, \& McClintock, 2012).

The growing interest in self-awareness, self-reflection, and transformation has extended beyond the field of education and is used in a variety of career fields (Chiaramonte \& Mills, 1993; Schön, 1983; Silverman, 2008; Stevens-Long et al., 2012). 
In education, the importance of self-awareness and self-reflection is not a novel concept and has been associated with experiential learning and student development (Ixer, 1999; Jordi, 2011; Lawrence-Wilkes \& Ashmore, 2014; Mezirow, 1991; Palmer \& Zajonc, 2010; Rosin, 2015; Schön, 1987). When working with students, educators are more acutely aware of the transitory state of a human being - ever changing, adapting, and transforming according to experiential, situational, relational, and social contexts. To understand the state of another person, while gaining insight into the self, educators must not only consider the relationship in place and context involved, but also have a developed sense of self-awareness (Chiaramonte \& Mills, 1993; Schön, 1983, 1987; Palmer \& Zajonc, 2010).

Educators in counseling, social work, healthcare, and other areas have incorporated the use of self-reflection and reflective techniques into their training programs (Ixer, 1999; Kinsella, 2007; Mezirow, 1991; Schön, 1983, 1987; Silverman, 2008; Stevens-Long et al., 2012). The term "self-reflection" has a range of definitions and associations and plays a key role in the learning process (Rosin, 2015; Schön, 1991). Theoretically, selfreflection and reflective practice account for the type of learning and development that extends beyond the confines of scientific and technological means of solving problems and resolving difficulties, which are found in the application process and exist as "problems of practice" (Kinsella, 2007, p. 103). Learning may consist of re-examining a particular meaning, providing a meaningful explanation for an event, or testing the validity of inner thoughts by taking action (Kinsella, 2007; Mezirow, 1991). Reflective learning may result in finding new meaning, confirming previous experiences, adding new knowledge, and obtaining insights that are more comprehensive (Kinsella, 2007; Mezirow, 1991; Schön, 1983). Mezirow (1991) believed that self-reflection is critical for intentional learning to occur. Chiaramonte and Mills (1993) defined self-reflection as a "deliberative and conscious process in which the person evaluates self image and self direction with a view of taking control of the way the self is shaped" (p. 145).

Literature exploring theoretical perspectives, methodical structure, and assessment of self-reflection is inconsistent and debatable. However, numerous authors see the potential benefits of self-reflection in student learning and professional development (Ixer, 1999; Kinsella, 2007; Lawrence-Wilkes \& Ashmore, 2014; Mezirow, 1991; Palmer \& Zajonc, 2010; Schön, 1983). For educators, the use of self-reflection and reflective practices can help them to better guide themselves through their personal experiences and improve their work. Self-reflection is a valuable tool that has the potential to be transformative in many disciplines and career fields. In counseling and mental health education, the practitioners' ability to be helpful and harmful is dependent on their own self-awareness and knowledge of themselves (Rosin, 2015; Schön, 1991; Stevens-Long et 
al., 2012). Implicit and explicit expectations, attributed characteristics from others, and the individual's "sense of self" contribute to the person's overall self-image and identity.

The ability to gain self-awareness and be reflective allows space for future growth and learning. Without the ability to self-reflect, character traits that are more individualistic, competitive, and manipulative can increase and become destructive (Chiaramonte \& Mills, 1993; Palmer \& Zajonc, 2010; Schön, 1983). For instance, without teaching the importance of self-reflecting, self-checking, and critically evaluating experiences, counselor educators may simply teach students to mimic behaviors that appear to be "effective." Thus, future counselors may lack the ability and therapeutic competency to consider negative implications, evaluate ethical guidelines, and resolve ambiguities and conflicting needs (Chiaramonte \& Mills, 1993; Schön, 1983, 1991; Silverman, 2008; Skovholt \& Jennings, 2005; Skovholt \& Rønnestad, 1992). While reviewing the potential benefits of self-reflection, Chiaramonte and Mills (1993) also warned against extreme forms of self-reflection and self-awareness. Much like other personal characteristics and abilities that may go unchecked, overemphasis of self-emphasis and self-reflection may lead to self-absorption and self-importance instead of reduction of self-ignorance.

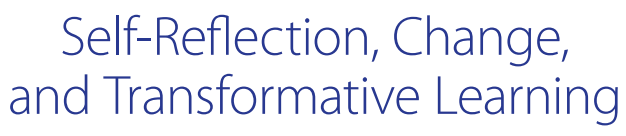

Associated with the concept of self-reflection and reflective practice is the notion of transformation and change. Literature addressing the topic of spirituality, religion, personal development, and adult education commonly mentions both self-reflection and transformative learning (Morgan, 2010; Palmer \& Zajonc, 2010; Schön, 1987). Transformative learning may involve change within a person's identity and sense of self (Illeris, 2014; Rosin, 2015). The terms transformative education and transformative learning vary slightly between authors and theories. Transformative learning may refer to the outcome caused by the change, the learning that occurred within the individual, and the educational method and program involved (Mezirow, 1991; Stevens-Long et al., 2012). In many cases, transformative learning is a term that refers to the "intra-psychic and/or behavioral process of a learner involved in a transformative experience-it is about what the learner does, feels, and experiences" (Stevens-Long et al., 2012, p. 184). The concept includes feelings of disorientation, critical reflection about personal experiences, examination of assumptions, and search for new meaning. Like the concept of self-reflection, transformative learning is connected to attaining a sense of universality, tapping into a spiritual and religious sense of being, and viewing the 
self in relation to other people and communities. Researchers believed that certain environmental dynamics and features promote change and transformative learning; however, they did not provide clear distinctive characteristics that contribute to transformative learning (Illeris, 2014; Morgan, 2010; Stevens-Long et al., 2012).

Transformation can generally occur in a variety of contexts and generate emotions of "universality" combined with an awareness of differences (Kottler, 2002; Morgan, 2010). The context can be a physical place or a psychological state (Morgan, 2010). Involvement in outdoor activities, experience of psychological stress and life dilemmas, and perplexity caused by changing environments will disrupt old routines and habits and increase the likelihood of personal development and transformation (Morgan, 2010; Palmer \& Zajonc, 2010; Stevens-Long et al., 2012). The amount of change and transformation that takes place is not solely dependent on external factors. Internal factors like the person's readiness and motivation will also influence his or her probability of having a transformative experience.

New and unfamiliar environments have the potential to stimulate transformation within individuals by alleviating monotony and challenging established routines (Kottler, 2002; Morgan, 2010; Stevens-Long et al., 2012). Kottler (2002) believed that unfamiliarity with a new environment might change people's mindset and allow individuals a chance to re-examine their perception of themselves and question preexisting worldviews. Ordinary routines that once worked in an accustomed environment may not work in a new setting. The unfamiliarity, confusion, and disorientation that are typically associated with moving to a new location can stimulate emotions that activate new, innovative responses and reactions that facilitate change (Kottler, 2002).

Activities like travelling to foreign geographical locations and experiencing new cultures can better activate an adequate amount of disorientation and discomfort to facilitate change and transformation (Morgan, 2010). Obtaining a deeper sense of purpose through participating in intercultural dialogue, protecting the environment, and volunteering to help people may improve an individual's chances of having a transformative experience (Palmer \& Zajonc, 2010). The external environment, along with a great sense of purpose, may potentially promote inner psychological growth within individuals (Morgan, 2010; Palmer \& Zajonc, 2010). The new setting disrupts established habits and challenges people's existing perspectives and worldviews and makes room for self-exploration and self-discovery. A new place and location offer new scenes and experiences that motivate people to develop new ways to live and survive. The characteristics of the new physical environment, in combination with the explorer's 
interactions, can either promote or hinder the chances of having a transformative experience (Kottler, 2002; Morgan, 2010; Palmer \& Zajonc, 2010).

Since emotional and intellectual struggles can also be experiential and have a transformative effect, normal life events, such as the death of a loved one, or strategic interventions, such as an educational and developmental program, may initiate change within a person (Morgan, 2010; Stevens-Long et al., 2012). Human difficulties and dilemmas can arise without any physical relocation. Sometimes certain landscapes, landmarks, nature, and the wilderness can hold special meaning and potentially have transformative powers. Consequently, even local places can provide new perspectives and experiences (Kottler, 2002; Morgan, 2010; Palmer \& Zajonc, 2010). Educational programs and activities that offer opportunities for small groups of people to gain new experiences and participate in self-reflection and reflective practices can facilitate change. Travel, contemplative experiences, communion with nature, and cross-cultural interaction can all be transformative.

\section{A Personal Reflection}

As a third-year doctoral student who has worked as a career development professional for more than 10 years, I have stressed the importance of self-reflection and experiential learning for others but neglected myself. Only recently has the prospect arisen for me to apply the same philosophy and principles to my own experience. As I reflect upon the changes in my life over the past three years, I am now able to see how random circumstances and situations contributed to changes in my personal and professional life. First, I made a decision to return to school and pursue a doctorate in counseling and counselor education degree. Second, I left a familiar role as a career services professional to become a training specialist, which involved relocating to a new university. Then, I participated in a study-abroad trip to Great Britain and gained a different perspective about my relationship with others and myself.

As a first-generation college student and an Asian-American woman who has grown up in the United States (US), I expected to see many similarities between the US and Great Britain. I anticipated minor language difficulties and did not worry about experiencing any culture shock. At the same time, I was aware that my personality could influence my interaction with group members on the trip and with the people whom I encounter. I am naturally quiet and reserved, while being able to be quite direct when needed. When meeting with new people, I am not very talkative. Besides exchanging typical 
greetings, I like to listen to people and ask few questions. In social settings, counseling sessions, and classrooms, I am comfortable when there is silence and think that it is "normal." In familiar environments, I have my regular, normal routines and go through each day without much thought. In new settings, however, I must consciously make an extra effort to adapt to the environment and interact with the people around me.

Since the US and Great Britain share a common language, I relied on my preexisting knowledge to communicate. However, when I visited Scotland, I was aware that my comfort level varied according to slight variations of the Scottish accents. As I listened to a group of seven people in Scotland talk about their approach to change and therapy, I noticed my ability to decipher certain words and phrases changed depending on the accents. This reminded me of regional differences that exist in the US and the variety of southern and northern dialects. In counseling and counselor education, the counselors' own language and background influences the ease with which they are able to understand and relate to their clients and students. I noticed that I had to be more attentive to accents less familiar to me. In a different situation, as I listened to a ticket salesperson give directions, I misheard the street "Knightsbridge" and mistakenly heard "Ninth Street." Later that night, I found myself thinking about how I heard "Ninth Street" instead of "Knightsbridge" when the salesperson tried to give directions. Since I was not familiar with "Knightsbridge," I had unintentionally converted an unfamiliar street to a common street name I knew in the US. I wondered how many times I had missed the true meaning in a conversation by inaccurately hearing and incorrectly translating something that was unfamiliar to me to something more familiar.

In another situation, the new environment, along with an unexpected event, caught me off guard and made me reconsider my normal routine. While on a train, I needed to use the "toilet" and walked to the back of the train. Once I got to the location, I opened the unlocked, rotating door to the restroom and was surprised by the site of a woman sitting on the toilet. After a brief exchange of shocked expressions, frantic movements, and shaken woman's departure, I had the opportunity to examine the interior of the stall and learned that the restroom door and toilet operated with different buttons. There were different buttons to operate the door and toilet, along with a special button to request help from personnel. Although I did not require any assistance and did not remember pressing the help button, two male employees greeted me when I exited the door. Once I told the two men that I did not need any assistance, they scurried away, perhaps sensing my embarrassment. In the moment, I felt embarrassed by the toilet incident. However, I realized later that these unexpected circumstances and unpredictable events help break established routines in learning environments and promote change. The unexpected experience reminded me to slow down before 
walking into an unfamiliar setting and showed me a different way of operating and functioning as a person.

The past three years shifted my view and perspective of the world. I learned to adapt to changes in circumstances and environments and grew as a professional. The trip to Great Britain reminded me of the value of professional development, importance of self-reflection, and significance of transformative education. After having time to reflect upon my experiences, I have a stronger connection to humanity and a better understanding of the human condition. As educators and human beings, we will continue to search for new passions, reinvent ourselves, and find our place in the universe. The opportunity to see and remember similarities and differences between cultures, peoples, and experiences, locally, nationally, and internationally reminded me of my personal choice to help others and work in higher education. Visiting with the people in Great Britain, experiencing people pass by right and left in Victoria Station, enjoying the scenery during the train rides between Scotland, Wales, and England, and looking out into the vast North Sea in Scotland made me feel "small" and "big" at the same time. My sense of time and space, view of unique individuals and group identity, knowledge of breadth and depth of cultural worldviews and way of life intersected like different train routes in Great Britain. I see myself as an ordinary person bound to others by universal struggles, a unique individual with my own personality and life experiences, a professional unbounded by traditional paths and specific positions, a contributor to society, and a global citizen.

\section{Implications}

Due to the variety of definitions and theories available, clarification of the term self-reflection is needed prior to the development of any new program. Educators can start the process by exploring details connected to the definitions of selfreflection and transformative learning. Choosing a particular model to help guide the reflective process and educational program could help to provide the structures needed. Applying Mezirow's three levels of learning and self-reflection that involve content, process, and premises may offer a more comprehensive understanding of the concept (Rosin, 2015). Having a clearer understanding of the self-reflection process and its applicability to teaching will help to guide the learning objectives for different activities and programs, which ultimately influences the learning outcome and growth opportunities. Transformative learning does not necessarily require international travel, a rigid program structure, or complete environmental control; therefore, educational 
activities and programs can be flexible and creative. Unpredictable experiences that program participants may encounter can contribute to their development and learning (Kottler, 2002; Morgan, 2010).

New environments and activities that create a sufficient amount of internal conflict and distress can offer openings for learning and change to occur. An educational program with a small group of people in an unfamiliar setting may adequately challenge the routine and daily comfort of living in the familiar. As mentioned earlier, educators need to monitor the level of anxiety and stress experienced by program participants and strategically include screening and support systems to optimize the chances for transformative learning. Assessing the participants' readiness by evaluating their cognitive and emotional ability to process their experiences and reviewing the appropriateness of the new environment based on the intended outcome may help to determine an individual's fit for a program. In addition, providing mentors, coaches, and collegial support to help participants cope with stress, develop new adaptive strategies, and obtain meaningful experiences can promote transformative learning (Kottler, 2002; Palmer \& Zajonc, 2010; Rosin, 2015).

\section{Conclusion}

A self-reflective, transformative experience is an existential and spiritual type of experience and should not be confused with an act of escape and denial of reality. The state of mind and intentionality of a traveler visiting a new country or new environment influences the quality of the experience, type of learning acquired, and meaning associated with the visit. The key to supporting transformative learning is to ensure that there is an adequate amount of disorientation and disturbance, along with sufficient relational support, to shift patterns of behaviors towards transformation (Kottler, 2002; Morgan, 2010; Palmer \& Zajonc, 2010; Stevens-Long et al., 2012). Too great a disorientation and disequilibrium may create too much anxiety and discomfort and interfere with transformation and growth. Undergoing internal conflicts, grappling with the process of self-questioning, and redefining an identity are common elements of the learning and growth process (Jordi, 2011; Palmer \& Zajonc, 2010; Rosin, 2015). The endeavor to learn and grow as individuals is a universal reality shared by everybody worldwide, regardless of their profession. 


\section{References}

Chiaramonte, P., \& Mills, A. J. (1993). Selfreflection counselling as an instrument in organisational learning. British Journal of Guidance and Counselling, 21(2), 145.

Freire, P., \& Freire, A. (1994). Pedagogy of hope: Reliving pedagogy of the oppressed. New York: Continuum.

Illeris, K. (2014). Transformative learning and identity. Journal of Transformative Education, 12(2), 148-163.

Ixer, G. (1999). There's no such thing as reflection. The British Journal of Social Work, 29(4), 513-527.

Jordi, R. (2011). Reframing the concept of reflection: Consciousness, experiential learning, and reflective learning practices. Adult Education Quarterly, 61(2), 181-197.

Kinsella, E. A. (2007). Technical rationality in Schön's reflective practice: Dichotomous or non-dualistic epistemological position. Nursing Philosophy, 8(2), 102-113.

Kottler, J. A. (2002). Transformative travel: International counselling in action. International Journal for the Advancement of Counselling, 24(4), 207-210.

Lawrence-Wilkes, L., \& Ashmore, L. (2014). The reflective practitioner in professional education. New York: Palgrave Macmillan.

Mezirow, J. (1991). Transformative dimensions of adult learning. San Francisco: Jossey-bass.

Morgan, A. D. (2010). Journeys into transformation: Travel to an "other" place as a vehicle for transformative learning. Journal of Transformative Education, 8(4), 246-268.
Palmer, P. J. (1998). The courage to teach: Exploring the inner landscape of a teacher's life. San Francisco: John Wiley \& Sons, Inc.

Palmer, P. J., \& Zajonc, A. (2010). The heart of higher education: $A$ call to renewal. San Francisco: Jossey-Bass.

Rosin, J. (2015). The necessity of counselor individuation for fostering reflective practice. Journal of Counseling and Development, 93(1), 88-95.

Schön, D. A. (1983). The reflective practitioner: How professionals think in action. New York: Basic Books, Inc.

Schön, D. A. (1987). Educating the reflective practitioner: Toward a new design for teaching and learning in the professions. San Francisco: Jossey-Bass Publishers.

Schön, D. A. (1991). The reflective turn: Case studies in and on educational practice. New York: Teachers College Press.

Silverman, E. (2008). Ongoing self-reflection. American Journal of Speech-Language Pathology, 17(1), 92.

Skovholt, T. M., \& Jennings, L. (2005). Mastery and expertise in counseling. Journal of Mental Health Counseling, 27(1), 13-18.

Skovholt, T. M., \& Rønnestad, M. H. (1992). Themes in therapist and counselor development. Journal of Counseling and Development, 70(4), 505-515.

Stevens-Long, J., Schapiro, S. A., \& McClintock, C. (2012). Passionate scholars: Transformative learning in doctoral education. Adult Education Quarterly, 62(2), 180-198. 


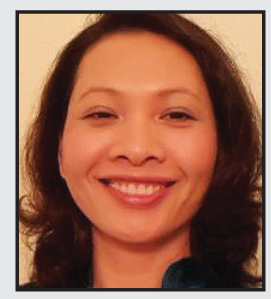

Vanden Thong is a PhD student in the Department of Education-Counseling and Counselor Education program at Texas Christian University. She received her Master of Education in Educational Psychology from The University of Texas at Austin. She has over nine years of career development experience, which includes five years of supervisory experience. Vanden has worked with a variety of individuals and groups experiencing career and work transition difficulties. Her research interests include individual and group resilience, systems and organizational dynamics, and solution-focused work. Presently, she is working as a trainer in a university setting. 